\title{
Dermis mechanical behaviour: influence of cell removal treatment
}

\author{
A. L. Audenino ${ }^{1}$, C. Bignardi ${ }^{1}$, E. Businaro ${ }^{1}$, C. Castagnoli $^{2}$, \\ M. Terzini ${ }^{1}$ \& E. M. Zanetti ${ }^{3}$ \\ ${ }^{1}$ Department of Mechanical and Aerospace Engineering, \\ Politecnico di Torino, Italy \\ ${ }^{2}$ Department of Plastic Surgery, Skin Bank, Trauma Center, Torino, Italy \\ ${ }^{3}$ Department of Industrial Engineering, University of Perugia, Italy
}

\begin{abstract}
The mechanical behaviour of skin is very important as regards dermatology, surgery and impact trauma. Engineered skin substitutes can bring significant medical benefit, in particular to patients with extensive burn wounds, even if current skin substitutes do not restore normal skin anatomy and its natural mechanical properties. This work considers the mechanical characterization of a particular layer of skin: dermis. Dermis can be used as a filling material and as support in different areas of reconstructive plastic surgery such as post mastectomy reconstructive surgery and abdominal surgery. The aim was to verify the influence of the decellularization treatment on its properties. The specimens were subjected to uniaxial static tests performed with Bose Electroforce ${ }^{\circledR} 3200$ and experimental data were represented with engineering and real time stress-strain curves. To begin, descriptive parameters were identified for stress vs. strain curves, such as ultimate tensile strength and maximum Young's modulus, and they were subsequently compared through multivariate analysis of variance to determine the influence of specimen cut orientation and decellularization treatment duration. Dermis, that had been decellularized over 5 or 6 weeks, exhibited mechanical properties comparable with natural ones and ultimate tensile strength and maximum Young's modulus were shown to be considerably higher in real time curves than in engineering ones.
\end{abstract}

Keywords: human dermis, static characterization, decellularization treatment. 


\section{Introduction}

The skin is the largest organ of the body; it works as a barrier to the environment and it controls thermal regulation and hydration retention. In order to be able to perform these critical functions the skin is constantly being renewed and it possesses the ability to autorepair wounds. Engineered skin substitutes have a significant medical practice in relation to patients with extensive burn wounds. However, current skin substitutes do not restore the full native skin physiology since they lack some components like hair follicles, sebaceous glands and sweat glands; besides, the engineered tissue cannot faithfully replicate the mechanical properties of normal skin.

Advances in tissues engineering behaviour let foresee that skin substitutes will be indistinguishable from the normal skin in a near future. Alloplastic material and skin allografts, taken from multi-organ donors, are the most suitable replacement integumentary for reconstructive surgery (Wong and Chang [1]). In fact, they maintain the peculiar architectural structure of the dermis and the decellularized human reticular dermis is a not immunogenic product when transplanted in the patient. Acellular reticular dermis grafting is therefore appropriate as a filling material and as support in different areas of reconstructive plastic surgery such as post mastectomy reconstructive surgery and in abdominal surgery.

The skin is made of three layers (epidermis, dermis, hypodermis) and it can be described as anisotropic, viscoelastic, nonlinear (Fung [2]) and nonhomogenous material; besides it has the ability to withstand large deformations. Both in vivo and ex vivo approaches have been developed to test these properties. Ex vivo tests allow to measure properties which cannot be studied in vivo. For instance, it is possible to perform destructive tests in order to determine the specimen failure mechanisms and its tensile strength. Furthermore, the tissue layers can be separated and evaluated independently.

The tensile test is the most widely used mechanical test performed on ex vivo skin specimens. Using this method, the anisotropic, non-linear and viscoelastic behaviours of skin have been explored, as well its failure (Pereira et al. [3]), creep (Del Prete et al. [4]), fatigue (Muñoz et al. [5]) and preconditioning behaviour (Liu and Yeung [6]). Another benefit of using ex vivo skin tissue is that changes in biomechanical behaviour, due to alteration of the skin's anatomical structure, can be assessed. This approach has been followed by those authors who varied the collagen content (Del Prete et al. [4]), or elastin and proteoglycans contents (Eshel and Lanir [7]) within the skin. In addition, ex vivo mechanical testing has a significant impact on the development of new engineered tissue, as it offers a reference or 'gold standard'. However, the mechanical properties of skin ex vivo result differently when compared with in vivo analysis because of the absence of pre-stress and hydration (Groves et al. [8]).

In general the dermis makes a major contribution to the overall mechanical characteristics of the skin because its main constituents are collagen and elastin fibrils allowing for high levels of deformation and flexibility, as the fibrils 
stretch and re-orientate (Sanders [9]). The overall mechanical response of the skin tissue under applied tension can be divided into three main phases (Holzapfel [10]) (shown in fig. 1). Each phase can be related to the collagen and elastin fibrils within the dermis. Phase one illustrates great extension at low load and Young's modulus as the dermal fibrils orientate towards the load axis. Phase two shows a gradual increase of the Young's modulus. Phase three is characterized by a region with maximum Young's modulus which remains relatively constant but finally decreases, culminating in the complete failure of the fibrils.

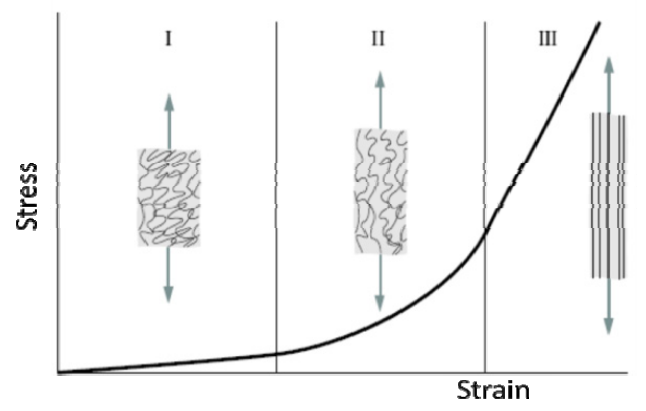

Figure 1: $\quad$ Schematic diagram of a typical tensile stress-strain curve for skin showing the associate collagen fiber morphology (Holzapfel [10]).

When at rest, the dermis is organized into a two-dimensional chaotic network without apparent manifestation of preferential directions of the fibrils' orientation; the area with a low Young's module is associated to the stretching of the waves in collagen fibrils normally present in the relaxed tissue and to their orientation along the direction of the load. As soon as the fibrils are aligned to the load direction they begin to withstand the applied force and the Young's modulus starts to increase. When all the fibrils are aligned the Young's modulus achieves the maximum value and it keeps constant as long as some fibrils yield, causing a progressive decrease of the Young's modulus and reaching complete rupture (Holzapfel [10]).

\section{Materials and methods}

\subsection{Specimens}

Strips of skin tissue, collected from donors back, were dissected along the craniocaudal direction and specimens were obtained cutting out the strips along craniocaudal (CC) and mediolateral (ML) directions. They were decellularized in $\mathrm{NaOH}$ culture. The skin bank provided specimens either non-chemically treated or incubated from 1 to 7 weeks, all preserved in a refrigerator with some milliliters of glycerol at $85 \%$. In order to decrease the number of factors that could bias results, the tests were carried out on a single donor, obtaining only 3-4 specimens for each combination of treatment duration and cut orientation. 


\subsection{Photographic set-up}

Two different photographic set-ups have been developed in order to size the specimens. The first one was finalized to measure the specimens' dimension at rest and was made of a full-frame digital camera (Canon EOS 5D Mark II), with an autofocus lens for macro photography (Canon EF 100mm f/2.8 Macro USM), a camera stand with two light stands and a tripod. A second set-up was developed in order to follow rupture tests in real time. It included the previously described digital camera, a second digital single-lens reflex camera (Canon EOS 400D), a tripod and remote capture software (DSLR Remote Pro).

Before testing, specimen pictures were taken from the top and from the side (fig. 2). The width and the thickness of the middle zone of the specimens were measured using the image analysis software ImageJ (as an average of five different measures).
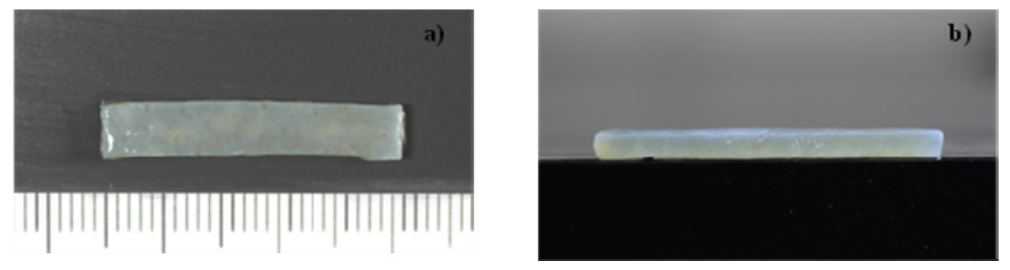

Figure 2: $\quad$ Specimens: a) top view; b) lateral view.

\subsection{Mechanical tests}

The specimens were washed in physiological solution to eliminate all the glycerol and were successively subjected to uniaxial tests in order to assess their mechanical properties (fig. 3); test parameters were selected according to natural tissue properties and Bose Electroforce ${ }^{\circledR}$ features. Each failure tensile test was performed in displacement control at a strain rate of $0.032 \mathrm{~s}^{-1}$.
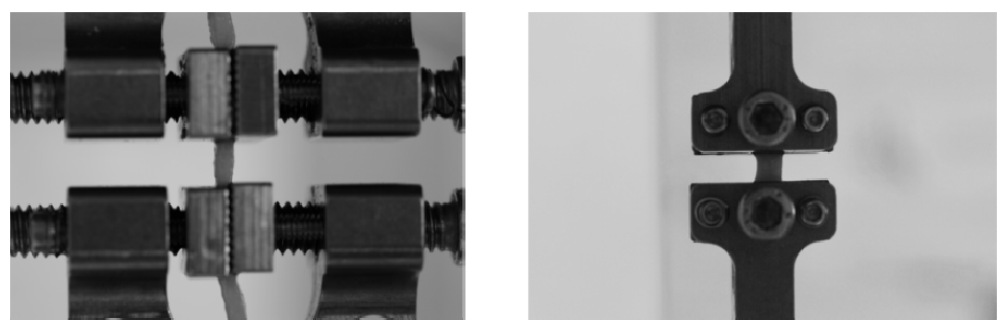

Figure 3: $\quad$ Specimen mounted onto the Bose Electroforce ${ }^{\circledR} 3200$. 


\subsection{Elaboration data}

In literature, the results of soft tissues failure tests have been reported following two different representations: some authors report 'engineering' curves (Annaidh et al. [11]), where stress and strain are calculated from load and displacement, referring to the initial specimen cross section and length, respectively. Other authors make use of 'real time' curves specimen cross section and length are measured in real time (Yoder and Elliott. [12]). In this work, both representations have been reported.

First of all some descriptive parameters have been identified in both curves: the ultimate tensile strength (UTS) and maximum Young's modulus (E). The normality of the statistical distribution of both parameters has been tested by lillietest function in Matlab R2010a; since this test gave a positive result multivariate analysis of variance (ANOVA) has performed in order to assess the influence of specimen cut orientation and duration of cell removal treatment. Significance levels for these tests were set to $\mathrm{p}<0.05$.

Moreover, ANOVA was followed by the Tukey-Kramer post-hoc test to compare the averages of two reference groups (non-chemically treated specimens cut along two directions) with the averages of each combination of duration treatment and specimen orientation.

\section{Results and discussion}

The results of uniaxial experimental tests were compared assuming a perfectly uniaxial loading condition, homogeneous stress-strain fields and a uniform distribution of collagen fibers.

Since in the literature a 'standardized way' to report the results of soft tissues failure tests has never been defined, engineering and real time curves have been here reported. Both formulations have produced a softening trend of stress-strain curves and a nonlinear elastic and an anisotropic behaviour of the tissue. Furthermore, the decellularization treatment has proven to reduce dermal mechanical properties, considering all incubation times. An incubation period between 5 and 6 weeks makes an exception since, in this case, the mechanical properties are partially recovered and this is demonstrated by the trends of the mechanical descriptive parameters such as ultimate tensile strength (UTS) and maximum Young's modulus (E) as shown in figs 4a) and 4b).

An overview of decellularization methods (Crapo et al. [13]) confirmed our results regarding $\mathrm{NaOH}$ cell removal treatment. In fact, it stated that bases (e.g. calcium hydroxide, sodium sulphide, and sodium hydroxide) are harsh enough and so are commonly used to eliminate growth factors from the matrix, even though they decrease ECM mechanical properties more significantly than chemical and enzymatic agents. The primary mechanism by which bases such as sodium hydroxide reduce mechanical properties is the cleavage of collagen fibrils and disruption of collagen crosslinks. Moreover, Richters et al. [14] also evaluated a cost-effective method based on low concentrations of $\mathrm{NaOH}$ for the 

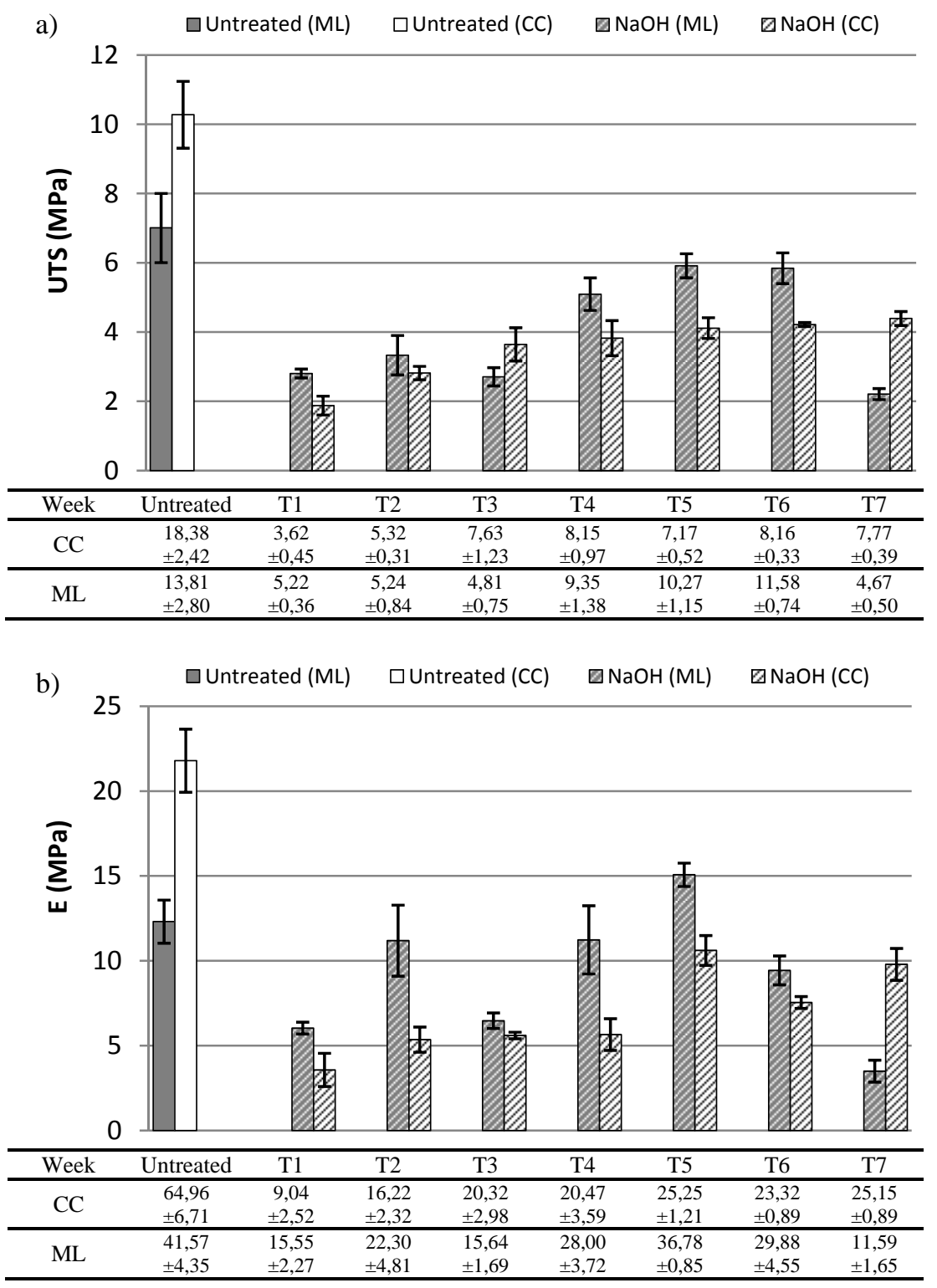

Figure 4: a) Engineering ultimate tensile strength (UTS) and b) engineering maximum Young's Modulus (E) with relative standard deviations in function of the period of treatment (the number following $\mathrm{T}$ represents the number of treatment weeks) for specimens cut along craniocaudal (CC) and mediolateral (ML) directions respectively. 
decellularization of human donor skin preserved in 85\% glycerol. They found that a 6-week incubation period was optimal, while longer periods caused damage to the collagen fibers, although elastin fibers appeared to be well preserved.

The influence of specimen orientations and treatment duration on the mechanical parameters (UTS, E) was evaluated applying statistical tests (ANOVA) to the results obtained from the engineering curves. This type of analysis turned out to be only qualitative because engineering curves were based on a simplified hypothesis that could not be considered adequate for soft tissue. As well known soft tissues specimens cross section undergoes significant changes, while very large displacements take place. ANOVA demonstrated how both specimen cut orientation and treatment duration have significant influence on UTS and E. Multiple comparison demonstrated how specimens cut along mediolateral direction and decellularized for 5 and 6 were not significantly different from 'untreated' ones, both considering UTS and E, with a confidence level of $95 \%$ and $97 \%$ respectively. On the contrary, all specimens cut along craniocaudal direction, for whatever treatment duration, were significantly different from the reference native group.

Since the engineering curves do not lead to quantitative significant values of the parameters, real time curves have been considered. Values so calculated are the most suitable to be used as an input to for finite element models. Even if the trend of the real time curves remain similar to the engineering ones, UTS and E values are considerably higher: $3 \mathbf{- 4}$ times and up to 7 times respectively for UTS and $\mathrm{E}$ (fig. 5a) and fig. 5b)).

In literature there is paucity of published articles dealing with the properties of human skin measured in vitro. The superiority of mechanical behaviour (both in terms of UTS and E) of specimens cut along mediolateral direction compared to the specimens cut along craniocaudal direction, is confirmed by Annaidh et al. [11]. Besides Young's modulus data here calculated fall within the variability of the allograft results as reported by Yoder and Elliott [12].

\section{Conclusion}

This work is part of a project focused on the mechanical characterization of acellular human dermal matrix. The measurement of the fundamental mechanical properties of the decellularized excised human skin is mandatory in order to be able to develop engineered materials for skin reconstruction, and to compare their performances with those of native skin. This work was focused on failure tests and was aimed to the assessment of the impact of different durations of cell removal treatment on specimen's structural performance or material behaviour. Specimen orientation has been taken into consideration because of tissue anisotropy.

As a result, the authors were able to prove how the human excised dermal matrix tissue, when decellularized under incubation culture in $\mathrm{NaOH}$ for 5-6 weeks, exhibits mechanical properties, which are comparable to the native one. 
a)

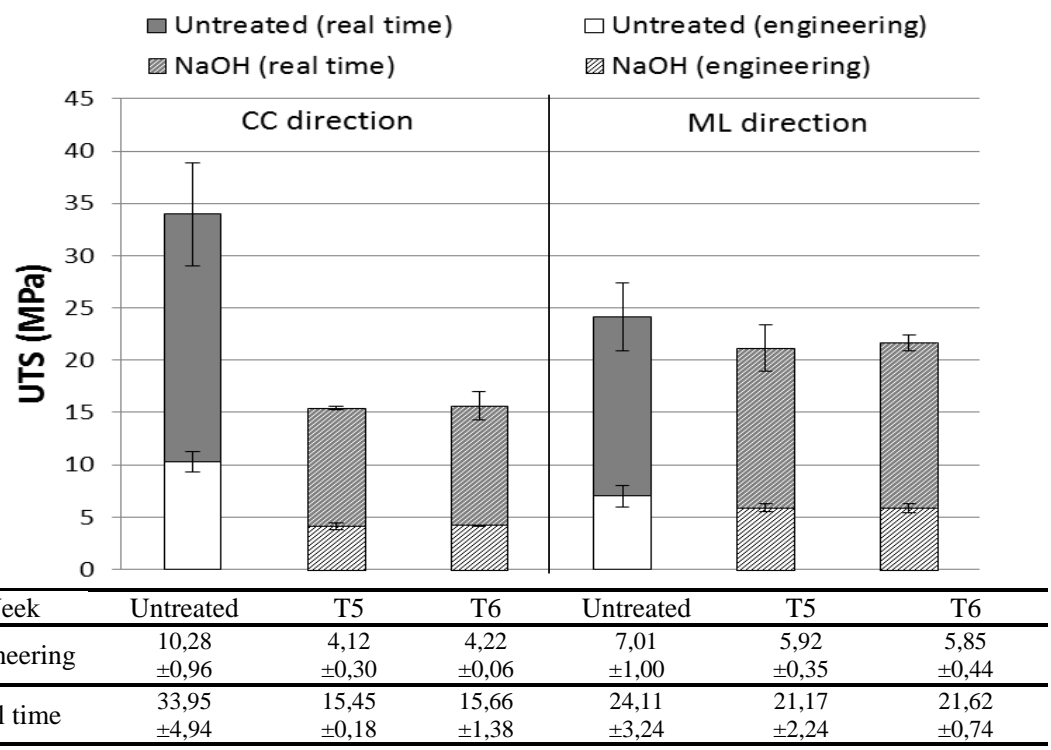

b)

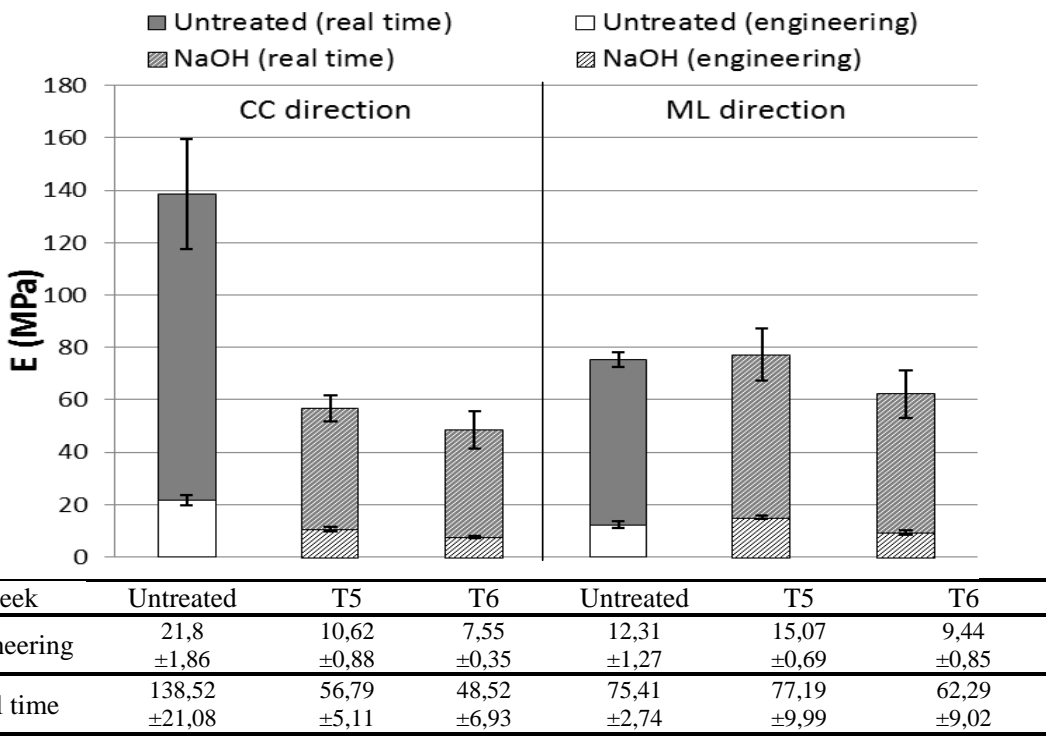

Figure 5: a) Comparison between ultimate tensile strength (UTS) values and b) maximum Young's modulus (E) values obtained for engineering and real time curves, with relative standard deviations, in function of the period of treatment (the number following $\mathrm{T}$ represents the number of treatment weeks) for specimens cut along craniocaudal (CC) and mediolateral (ML) directions respectively. 
Besides, the mechanical behaviour of specimens cut along the mediolateral direction was systematically higher than those cut along the craniocaudal one.

The values of ultimate tensile strength and maximum Young's modulus can be greatly underestimated whenever engineering stress/strain curves are considered; therefore real time stress/strain curves have been considered in order to better quantify the actual values of these parameters. These realistic values should be used in order to set up and validate skin computational models, such as those based on finite elements.

On the whole, indications about the optimal decellularization treatment duration have been given together with refined mechanical properties of the tissue.

\section{References}

[1] Wong, D.J. and Chang, H.Y., Skin tissue engineering, StemBook, ed. The Stem Cell Research Community, StemBook, 2009.

[2] Fung, Y.C., Biomechanics: Mechanical Properties of Living Tissue, Springer-Verlag: New York, 1993.

[3] Pereira, B.P., Lucas, P.W. and Teoh, S.H., Ranking the fracture toughness of thin mammalian soft tissue using the scissor cutting test. Journal of Biomechanics, 30(1), pp. 91-94, 1997.

[4] Del Prete, Z., Antoniucci, S., Hoffman, A.H., and Grigg, p.,Viscoelastic properties of skin in Mov-13 and Tsk mice. Journal of Biomechanics, 37 (issue 10 October), pp. 1491-1497, 2004.

[5] Muñoz, M.J, Bea, J.A, Rodríguez, J.F, Ochoa, I., Grasa, J., Pérez del Paloma, A., Zaragoza, P., Osta, R. and Doblaré, M., An experimental study of the mouse skin behaviour: Damage and inelastic aspects. Journal of Biomechanics, 41(1), pp. 93-99, 2008.

[6] Liu Z. and Yeung K., Preconditioning and stress relaxation of skin tissue. Journal of Biomedical \& Pharmaceutical Engineering, 2(1), pp. 22-28, 2008.

[7] Eshel, H. and Lanir, Y., Effects of strain level and proteoglycan depletion on preconditioning and viscoelastic responses of rat dorsal skin. Annals of Biomedical Engineering, 29(2), pp. 164-172, 2001.

[8] Groves, R.B.,Coulman, S.A., Birchall, J.C. and Evans, S.L., Quantifying the mechanical properties of human skin to optimise future microneedle device design. Computer Methods in Biomechanics and Biomedical Engineering, 15(1), pp. 73-82, 2012.

[9] Sanders, R., Torsional elasticity of human skin in vivo. Pflügers Archiv European Journal of Physiology, 342, pp. 255-260, 1973.

[10] Holzapfel G.A., Biomechanics of soft tissue. In Handbook of Material Behavior Nonlinear Models and Properties, eds Jean Lemaitre, LMTCachan, France, Academic Press, 2000.

[11] N. Annaidh, A., Bruyère, K., Destrade, M., Gilchrist, M. D. and Otténio, M., Characterization of the anisotropic mechanical properties of excised 
192 Modelling in Medicine and Biology X

human skin. Journal of the Mechanical Behaviour of Biomedical Materials, 5(1), pp. 139-148, 2012.

[12] Yoder J.H. and Elliott D.M., Non linear and anisotropic tensile properties of graft materials used in soft tissue applications. Clinical biomechanics, 25(4), pp. 378-382, 2010.

[13] Crapo, P.M., Gilbert, T.W. and Badylak, S.F., An overview of tissue and whole organ decellularization processes. Biomaterials, 32(12), pp. 32333243, 2012.

[14] Richters, C.D., Pirayesh, A., Hoeksema, H., Kamperdijk, E.W., Kreis, R.W. and Dutrieux, R.P., Development of a dermal matrix from glycerol preserved allogeneic skin. Cell tissue bank, 9(4), pp. 309-315, 2008. 